HDL-cholesterol, triglyceride and insulin concentrations. J Int Med 231: 25-30

7. Fuh MT-M, Jeng C-Y, Mason MS-S, Sheu WH-H, Chen YDI, Reaven GM (1993) Insulin resistance, glucose intolerance, and hyperinsulinemia in patients with microvascular angina. Metabolism 42: 1090-1092

8. Young MH, Jeng C-Y, Sheu WH-Het al. (1993) Insulin resistance, glucose intolerance, hyperinsulinemia and dyslipidemia in patients with angiographically demonstrated coronary artery disease. Am J Cardio 72: 458-460

9. Hollenbeck CB, Chen N, Chen Y-DI, Reaven GM (1984) Relationship between the plasma insulin response to oral

\section{Selection of control subjects for case/control analysis of susceptibility to Type 1 (insulin-dependent) diabetes mellitus}

\section{Dear Sir,}

Pozzilli et al. [1] suggest several possible sources for control subjects for studies of Type 1 (insulin-dependent) diabetes. For the examination of HLA-associations alone they suggest that blood donors are likely to be satisfactory, subject to adequate matching by ethnic group, which seems a very sensible and practical proposal if the donors' ethnicity is adequately known, an if the cases are obtained from the entire catchment area of the blood donor service.

They suggest, for studies looking at environmental factors, a number of additional sources for control subjects. They are of the view that individuals who may later develop diabetes should be excluded, and that various efforts should be made to match diabetic cases and control subjects for some of the known HLA associations.

We do not agree with this proposal. The purpose of matching in analytical epidemiological studies of environmental determinants of disease is quite different, and has nothing to do with the fundamental design issue of collecting diabetic cases and sampling control subjects in an unbiased way. Matching is essentially a convenience, and should be used for clearly identified, and easily measured risk factors, such as age and sex, which are not in themselves of interest as analytical variables. Briefly, matching induces a confounding of the disease-exposure relationship by the matching factors, and this confounding is adjusted for in the analysis. A consequence of this is that it is not possible to carry out any meaningful analyses using the matching factors. Indeed matching by HLA phenotypes would exclude the possibility of investigating the interaction between environmental exposures and genetic susceptibility.

The most significant development in epidemiologic methodology in the last decade is a more detailed understanding of the nature of the case-control study. Miettinen [2] has summarised this work, albeit rather opaquely. Rothman [3] is a more accessible source. The key notion is the unbiased comparison of a census of cases with a sample from a base of control subjects. This base can be most elegantly defined as "all those persons

Corresponding author: Dr. A. Staines, LRF Unit for Clinical Epidemiology, 17 Springfield Mount, University of Leeds, Leeds LS2 9NG, UK glucose and insulin stimulated glucose utilization in normal subjects. Diabetes 33: 460-463

10. Harano Y, Hidaka H, Takatsuki S et al. (1978) Glucose, insulin and somatostatin infusion for the determination of insulin sensitivity in vivo. Metabolism 27: 1449-1452

11. Greenfield MS, Doberne L, Kraemer F, Tobey T, Reaven GM (1981) Assessment of insulin resistance with the insulin suppression test and the euglycemic clamp. Diabetes 30 : 387392

who, if they were to develop the disease of interest during the study period, would be eligible to be cases" [3]

The choice of control subjects is critical. It is not difficult to match cases and control subjects too closely, and one of the proposals, selecting control subjects from classmates, would almost certainly accomplish this. There is good evidence [4] that factors acting in early life are important in the aetiology of childhood diabetes, and some evidence [5, and Staines, Bodansky, McKinney et al. unpublished observations] that area of residence is a significant risk factor for Type 1 diabetes. Selection of classmates would almost certainly match closely on residence, and might well match closely for social factors, which are related to infant feeding and child rearing practices. The potential influence of these factors could be entirely obscured.

Eliminating persons with a family history of diabetes from consideration as control subjects would tend to selectively eliminate families exposed to the putative environmental risk factors, and could therefore tend to exaggerate any associations which might be uncovered. It would also tend to strengthen any confounding present in the study population, and would render the entire analysis suspect. In additon the difficulty of implementing this in practice, and incurring a significant extra cost in approaching control subjects should not be under-estimated. A more appropriate approach would be to record information on family history, and to adjust for this in the analysis.

We agree that selecting two haplotype different siblings of diabetic patients as control subjects would cause serious problems of interpretation, both for the analysis of genetic risks, and for the analysis of environmental factors.

In conclusion the choice of appropriate control subjects for a case-control study is certainly not easy. Control subjects and diabetic cases should have precisely the same eligibility criteria, and control subjects should be sampled from the best available population lists, if these exist. If they don't, great care needs to be taken to avoid bias and overmatching of diabetic cases and control subjects. For case-control studies looking at environmental risk factors, (as opposed to cohort studies) it is hard to see any justification for genetic exclusion criteria applied to control subjects alone.

Yours sincerely,

A. Staines,

P. A. McKinney

\section{References}

1. Pozzilli P, Buzzetti R, Bottazzo GF, Tosi R (1993) The selection of control subjects for case/control analysis of susceptibility to type 1 (insulin-dependent) diabetes mellitus. Diabetologia 36: 1208-1209 
2. Miettinen OS (1985) Theoretical epidemiology. John Wiley, New York

3. Rothman K (1986) Modern epidemiology. Little Brown, Minnesota

4. Dahlquist G, Blom L, Lönnberg G (1991) The Swedish childhood diabetes study - a multivariate analysis of the de-

\section{Response from the authors}

\section{Dear Sir,}

We read with interest the letter of Drs. Staines and McKinney and we agree with their comments. However, in approaching the problem of selection of case/control analysis of Type 1 diabetes susceptibility in respect to environmental factors they do not agree with us in matching cases and control subjects for some of the HLA associations.

Corresponding author: Dr. P. Pozzilli, Università di Roma "La Sapienza", Istituto di Clinica Medica II, Policlinico Umberto I, Viale del Policlinico, I-155 00161 Rome, Italy terminants for diabetes in different age groups. Diabetologia 34: 757-672

5. Staines A, Bodansky HJ, Stephenson CR, Lilley H, Cartwright R (1992) Infection and the causes of childhood diabetes: evidence from community lifestyle studies. Diabetologia 35 [Suppl]: A24 (Abstract)

The question is whether one is interested in HLA mediated genetic susceptibility to Type 1 diabetes or not. In the former case it is clearly important not to match for HLA; however, in the latter case interest focuses on non-HLA genetic factors and therefore diabetic cases and control subjects should be matched for some of the known HLA phenotypes. Such HLA matching may also be necessary if there is an interaction between the hypothetical additional factor and a given HLA phenotype.

As the search for genes predisposing to Type 1 diabetes outside the HLA region is nowadays the subject of intense research, particular attention should be given when selecting control subjects as much as possible matched to patients for all known HLA DR and DQ associations.

P Pozzilli, R Buzzetti, GF Bottazzo, R Tosi

\section{Announcement}

Animal NIDDM and/or Obesity - Endogenous and Environmental Factors Involved in the Regulation and Pathogenesis.

This symposium will be held on 27 September 1994 in the SAS Royal Scandinavian Hotel, Düsseldorf, Germany, immediately before the EASD Meeting.

For further information please contact: Dr. L.Herberg, Diabetes Research Institute at the University of Düsseldorf,
Auf'm Hennekamp 65, D-40225 Düsseldorf, Germany, Tel.: + 492113382649 ; Fax: + 492113382603

or

Dr G.Löffler, Institute for Biochemistry, Genetics and Microbiology, University of Regensburg, Universitätsstrasse 31, D-93040 Regensburf, Germany. Tel.: + 499419432472 ; Fax: $+499419432474$ 